\title{
Editorial: Biology, Systematics, Taxonomy, and Evolution of Insect Vectors
}

\author{
Kaio Cesar Chaboli Alevi ${ }^{1,2 *}$, Cleber Galvão ${ }^{2}$, Mauro Toledo Marrelli ${ }^{1}$, \\ André Barretto Bruno Wilke ${ }^{3}$, Wanzhi Cai ${ }^{4}$ and Jader de Oliveira ${ }^{1}$ \\ ${ }^{1}$ Laboratório de Entomologia em Saúde Pública, Departamento de Epidemiologia, Faculdade de Saúde Pública, \\ Universidade de São Paulo (USP), São Paulo, Brazil, ${ }^{2}$ Laboratório Nacional e Internacional de Referência em Taxonomia de \\ Triatomíneos, Instituto Oswaldo Cruz (FIOCRUZ), Rio de Janeiro, Brazil, ${ }^{3}$ Laboratory for Computational Epidemiology and \\ Public Health, Department of Epidemiology and Biostatistics, Indiana University School of Public Health, Bloomington, IN, \\ United States, ${ }^{4}$ MOA Key Lab of Pest Monitoring and Green Management, Department of Entomology, College of Plant \\ Protection, China Agricultural University, Beijing, China
}

Keywords: biology, systematics, taxonomy, evolution, insects vectors

\section{Editorial on the Research Topic}

\section{Biology, Systematics, Taxonomy, and Evolution of Insect Vectors}

Vector insects comprise several hematophagous invertebrate species grouped in the orders Diptera, Hemiptera, Anoplura, and Siphonaptera (Kuno and Chang, 2005). These species are responsible for the transmission of many infectious diseases such as dengue, chikungunya, Zika, yellow fever, malaria, lymphatic filariasis, leishmaniasis, Chagas disease, sleeping sickness, onchocerciasis, bubonic plague, Rift Valley fever, Japanese encephalitis, West Nile fever, tungiasis, typhus, louse-borne relapsing fever, sandfly fever, Crimean-Congo hemorrhagic fever, Lyme disease,

\section{OPEN ACCESS}

Edited and reviewed by: Rodney L. Honeycutt, Pepperdine University, United States

*Correspondence: Kaio Cesar Chaboli Alevi kaiochaboli@hotmail.com

Specialty section:

This article was submitted to Phylogenetics, Phylogenomics, and Systematics,

a section of the journal

Frontiers in Ecology and Evolution

Received: 20 January 2022 Accepted: 24 January 2022 Published: 01 March 2022

Citation: Alevi KCC, Galvão C, Marrelli MT, Wilke $A B B$, Cai W and Oliveira J (2022) Editorial: Biology, Systematics,

Taxonomy, and Evolution of Insect Vectors. Front. Ecol. Evol. 10:858886.

doi: $10.3389 /$ fevo.2022.858886 relapsing fever, spotted fever, Q fever, and hence are of great importance to public health (World Health Organization, 2022). Vector-borne diseases account for more than $17 \%$ of all infectious diseases (caused by parasites, bacteria, or viruses), causing more than 700,000 deaths annually (World Health Organization, 2022). A better understanding of insect vector biology, systematics, taxonomy, and evolution can support more effective management and control strategies to mitigate the mortality and morbidity of these diseases. This is of great importance because, for the most part, vector control is the primary means by which incidences of vector-borne diseases in humans and animals are mitigated.

The studies presented in this Research Topic focused on two important orders of vector insects, namely, Diptera and Hemiptera. The genus Aedes Meigen, 1818 (Diptera, Culicidae) was discussed in several works: Martínez et al.; David et al.; Kelly et al.; Wang et al.; Virgínio et al., contributing to the taxonomic (Martínez et al.; Virgínio et al.), genetic/genomic (Martínez et al.; Kelly et al.), biological (David et al.) and entomo-epidemiological knowledge (Martínez et al.; Kelly et al.) of these vectors.

Martínez et al. presented important taxonomic, genetic (demonstrating that barcodes based on the COI gene fragment are successful method for identifying Aedes species) and entomoepidemiological [diagnosing natural infection for dengue virus type 1 (DENV-1) and chikungunya virus (CHIKV)] for Ae. aegypti (Linnaeus, 1762) and Ae. albopictus (Skuse, 1894) from the eastern region of Colombia.

David et al. evaluated the development of Ae. aegypti in response to different variables of the physicochemical of water. They observed a positive relationship between factors such as the abundance of immatures and container volume and concentration of dissolved organic carbon (DOC), average dry weight of females and wing lengths of males and females, and temperature, as 
well as between male mean dry weight and water conductivity. Furthermore, Wang et al. evaluated biotic (bacterial diversity) and abiotic (physicochemical factors) issues that influence the larval density of mosquitoes of the genera Aedes, Culex Linnaeus, 1758, Anopheles Meigen, 1818, and Mansonia Blanchard, 1901 in Jiaxiang County, Shandong Province, China and concluded that the density and species can be jointly affected by these evaluated factors.

Kelly et al. performed population genomics studies of Ae. aegypti and suggested that populations from Exeter, California (CA), were reintroduced from the southern CA cluster following the elimination of the vector in 2015 by Delta Vector Control District (DVCD) activities. Demari-Silva et al. evaluated the genetic structure of Anopheles cruzii Dyar and Knab, 1908 from Ribeira Valley, southeastern Brazil, and verified whether the genetic structure was associated with forest cover, elevation, slope, and vegetation physiognomy. The authors observed two distinct lineages in the studied region associated with elevation and isolation by distance and proposed that differences in observed population structure found might be associated with the distribution of bromeliad species. Virgínio et al. presented the wing image database of mosquitoes (WingBank), developed from the geometric morphometry of the wings of these vectors. This image bank contains data referring to 77 species belonging to 15 genera of Culicidae.

Studies of Chagas disease vectors are related to the Rhodniini tribe (Hemiptera, Triatominae) (Marchant et al.; Filée et al.; de Paula et al.). Marchant et al. performed a transcriptomic study to evaluate the chemosensory gene expression of Rhodnius robustus Larrousse, 1927 and R. prolixus Stål, 1859. Among the 199 transcripts annotated in this study, 93\% were conserved between $R$. prolixus and the sylvatic $R$. robustus; 12 novel takeout transcripts from the $R$. prolixus genome (Mesquita et al., 2015) were annotated; a large set of transcripts were found to be differentially expressed between males and females, with a majority of them overexpressed in males; 10 transcripts were specifically expressed in one sex and absent in another; three chemosensory transcripts were found to be expressed only in the reared $R$. prolixus and only one in sylvatic $R$. robustus; and 8,596 transcripts were differentially expressed, with most overexpressed in the sylvatic $R$. robustus samples.

de Paula et al. and Filée et al. performed phylogenetic [using mitochondrial rDNA (16S), nuclear ribosomal RNA (28S) and wingless $(\mathrm{Wg})$ sequences] and phylogenomic studies [using 15

\section{REFERENCES}

Kuno, G., and Chang, G. -J. J. (2005). Biological transmission of arboviruses: reexamination of and new insights into components, mechanisms, and unique traits as well as their evolutionary trends. Clin. Microbiol. Rev. 18, 608-637. doi: 10.1128/CMR.18.4.608-637.2005

Mesquita, R. D., Vionette-Amaral, R. J., Lowenberger, C., RiveraPomar, R., Monteiro, F. A., Minx, P., et al. (2015). Genome of mitochondrial genes $(13.3 \mathrm{~kb})$, partial nuclear rDNA (5.2 kb) and 51 nuclear protein-coding genes $(36.3 \mathrm{~kb})]$, respectively, in the Rhodniini tribe. de Paula et al. presented dating related to the origin of the tribe Rhodniini (17.91 Mya ago), of the genus Rhodnius (9.13 Mya ago) and observed a close relationship between Rhodniini and Salyavatinae, highlighting that these vectors diverged from this subfamily 30.43 Mya. Furthermore, the authors highlighted that the colonization of bromeliads, palms trees, and bird nests represent important events for the speciation of Rhodnius spp. and discussed the evolution of hematophagy in a scenario where Rhodniini's ancestor could be pre-adapted for the invasion of these ecotopes that presented favorable factors such as water availability, thermal damping, and source of food (vertebrate inquilines) for the species of Rhodniini. Filée et al. discussed taxonomic and systematic issues of the Rhoniini tribe. They confirmed the specific status of $R$. montenegrensis Rosa et al., 2012 and $R$. marabaensis Souza et al., 2016 and the synonymy of $R$. taquarussuensis Rosa et al., 2017 with $R$. neglectus Lent, 1954, questioned the specific status of $R$. milesi Carcavallo et al., 2001 (that is more likely $R$. nasutus Stål, 1859) and suggested the generic reclassification of Psammolestes tertius Lent and Jurberg, 1965 to $R$. tertius. The authors observed a greater relationship between pictipes and pallescens groups than with the prolixus group and discussed which introgression events occurred in the three vector groups.

Finally, $\mathrm{Wu}$ et al. carried out studies on egg cannibalism of Arma custos (Fabricius, 1794) (Hemiptera: Asopinae) and observed that females exhibit a higher tendency for egg cannibalism than males and that cannibalism varies not only with the developmental stage of the eggs and nymphs but also with sex and reproductive status of females.

Thus, the Research Topic Biology, Systematics, Taxonomy, and Evolution of Insects Vectors addressed an important gap in the knowledge associated with biological, systematic, taxonomic, and evolutionary processes of insect vector species and helps further our understanding of issues related to the dynamics of vector-borne disease transmission, thus providing direction for activities related to vector control programs.

\section{AUTHOR CONTRIBUTIONS}

All authors listed have made a substantial, direct, and intellectual contribution to the work and approved it for publication. 
Conflict of Interest: The authors declare that the research was conducted in the absence of any commercial or financial relationships that could be construed as a potential conflict of interest.

Publisher's Note: All claims expressed in this article are solely those of the authors and do not necessarily represent those of their affiliated organizations, or those of the publisher, the editors and the reviewers. Any product that may be evaluated in this article, or claim that may be made by its manufacturer, is not guaranteed or endorsed by the publisher.

Copyright (c) 2022 Alevi, Galvão, Marrelli, Wilke, Cai and Oliveira. This is an open-access article distributed under the terms of the Creative Commons Attribution License (CC BY). The use, distribution or reproduction in other forums is permitted, provided the original author(s) and the copyright owner(s) are credited and that the original publication in this journal is cited, in accordance with accepted academic practice. No use, distribution or reproduction is permitted which does not comply with these terms. 